\title{
Longitudinal wakes of a short magnet, coherent transition radiation, and coherent diffraction radiation for an ultrarelativistic electron bunch
}

\author{
R. A. Bosch \\ Synchrotron Radiation Center, University of Wisconsin-Madison, 3731 Schneider Dr., Stoughton, Wisconsin 53589, USA \\ (Received 18 February 2008; revised manuscript received 18 June 2008; published 31 October 2008)
}

\begin{abstract}
The longitudinal wake is calculated for a bunch of ultrarelativistic electrons that is deflected by a magnet that is much shorter than the formation length of its coherent synchrotron radiation. Using this wake and the Coulomb field of an image bunch, we obtain formulas for the wake of coherent transition radiation. By modeling the coherent diffraction radiation of an iris as the coherent transition radiation from a hollow bunch, we calculate the on-axis longitudinal wake of coherent diffraction radiation for an arbitrary target shape. We also calculate the influence of an upstream magnet's edge radiation upon the wake.
\end{abstract}

DOI: 10.1103/PhysRevSTAB.11.100704

PACS numbers: 41.60.Ap, 52.38.Kd

\section{INTRODUCTION}

The energy profile of a bunch of ultrarelativistic electrons is affected by the longitudinal wake of a bending magnet [1-4] or a diffraction-radiation target [5-7]. We calculate the longitudinal wakes of a short bending magnet, coherent transition radiation, and coherent diffraction radiation by relating them to the wake of suddenly accelerated electrons $[8,9]$. Each wake is the sum of a coherentradiation wake and the longitudinal space charge (LSC) wake of a bunch in uniform motion [10].

The wake of suddenly deflected electrons models a magnet that is short compared to the formation length of its steady-state coherent synchrotron radiation (CSR). The coherent-radiation wake is the wake of electrons that are stopped at the magnet entrance (the "entrance-edge wake") summed with the coherent-radiation wake of electrons accelerated in a different direction at the magnet exit (the "exit-edge wake") [11-13].

For a weak short magnet with deflection angle $\theta \ll 1 / \gamma$, where $\gamma$ is the relativistic factor, the entrance-edge wake of a bunch with rms length $\sigma_{z}$ moves ahead of the bunch a distance $\ll \sigma_{z}$ within the radiation formation zone. The exit-edge wake, which moves with the bunch in the formation zone, is nearly canceled by the entrance-edge wake. The coherent-radiation wake is a steady-state wake that is much smaller than the LSC wake.

For a strong short magnet with $\theta \gg 1 / \gamma$, the entranceedge wake overtakes the bunch within the formation zone, at a distance $\sim \sigma_{z} /(1-\cos \theta)$ downstream of the magnet, where the coherent-radiation wake changes from a steadystate wake to the resistive wake of exit-edge radiation.

By considering an image bunch, we relate coherent transition radiation to suddenly deflected electrons. When a bunch passes through a target at a nongrazing angle, the wake of forward transition radiation equals the wake of suddenly accelerated electrons, while the wake of backward transition radiation affects the bunch within a distance $\sim \sigma_{z}$ upstream of the target.
By modeling coherent diffraction radiation from a circular iris as the coherent transition radiation from a hollow bunch, we obtain the on-axis longitudinal wake of coherent diffraction radiation for an arbitrary target shape. The influence of an upstream bending magnet's edge radiation upon the wake is also calculated.

We calculate the coherent-radiation wakes within the radiation formation zone of length $\sim \sigma_{z} \gamma^{2}$ [2,3,8,9]. For bunch radius $r_{b} \ll \sigma_{z} \gamma$, the coherent-radiation wake may exceed the LSC wake near to the magnet or target, but the LSC wake dominates at the boundary of the formation zone and beyond [Ref. [9], Eq. (14)]. If one applies the formation-zone formulas at distances $\gtrsim \sigma_{z} \gamma^{2}$, the error is smaller than the LSC wake.

\section{SHORT MAGNET WAKE}

\section{A. Wake contribution from an electron}

Consider an ultrarelativistic bunch with radius $r_{b} \ll$ $\sigma_{z} \gamma$ that travels parallel to the $z$ axis after being deflected through a positive angle $\theta$. The contribution to the longitudinal on-axis wake from the sudden acceleration of an electron at distance $r$ from the $z$ axis at time $t=0$ is [9]

$$
\begin{aligned}
\tilde{E}_{\text {exit }}(r, z, \omega)= & \int_{-\infty}^{\infty} E_{\text {exit }}(r, z, t) e^{i \omega t} d t \\
= & \left(-\frac{e^{i k z} i q \omega}{2 \pi \varepsilon_{0} c^{2} \gamma^{2}}\right) K_{0}\left(\frac{|\omega| r}{c \gamma}\right) \\
& -\frac{e^{i k \sqrt{z^{2}+r^{2}}} q}{2 \pi \varepsilon_{0} c \sqrt{z^{2}+r^{2}}},
\end{aligned}
$$

for $r \ll z \ll \lambda \gamma^{2} / 2 \pi$. Here, $q<0$ is the electron charge, $\omega$ is angular frequency, $c$ is the speed of light, $k=$ $\omega / c$ is the wave number, $\lambda$ is the wavelength, $\varepsilon_{0}$ is the permittivity of free space, and $z$ is the distance downstream of the magnet. The first term on the right-hand-side of Eq. (1) is the longitudinal Coulomb field of an electron 
in uniform motion, while the second term is exit-edge radiation [9].

In Appendix A, we approximate the longitudinal field from an electron that is suddenly stopped after traveling at an angle $\theta$ with respect to the $z$ axis as

$$
\tilde{E}_{\text {ent }}(r, z, \omega)=\frac{e^{i k \sqrt{z^{2}+r^{2}} \cos \theta} q}{2 \pi \varepsilon_{0} c \sqrt{z^{2}+r^{2}}},
$$

for $z \ll \lambda \gamma^{2} / 2 \pi$ and $z \sin \theta \ll \lambda \gamma / 2 \pi$. Equation (2) describes the entrance edge radiation for $z \gg r$ when $\theta \leq$ $\pi / 2$, and for $z \gg r / \sin \theta$ when $\pi / 2<\theta \ll \pi-1 / \gamma$. These conditions may be written as $(\pi-\theta) / 2 \gg 1 / \gamma$ and $z \sin [(\pi-\theta) / 2] \gg r$.

For $z \gg r$, Eq. (2) describes a plane wave propagating in the direction of the bunch's initial motion. A deflected electron falls behind such a plane wave, so that a deflected bunch falls behind the entrance-edge wake. For $\theta \gg 1 / \gamma$ and $z \gg r / \theta$ [i.e., $z \sin (\theta / 2) \gg r$ ], $\tilde{E}_{\text {ent }}$ nearly equals the Coulomb field of an electron that travels indefinitely in the direction of the bunch's initial motion.

The on-axis formation-zone longitudinal field is the sum of Eqs. (1) and (2):

$$
\begin{aligned}
\tilde{E}(r, z, \omega)= & \frac{-q}{2 \pi \varepsilon_{0} c \sqrt{z^{2}+r^{2}}}\left(e^{i k \sqrt{z^{2}+r^{2}}}-e^{i k \sqrt{z^{2}+r^{2}} \cos \theta}\right) \\
& -\left(\frac{e^{i k z} i q \omega}{2 \pi \varepsilon_{0} c^{2} \gamma^{2}}\right) K_{0}\left(\frac{|\omega| r}{c \gamma}\right) .
\end{aligned}
$$

\section{B. Deflected bunch}

Consider a bunch of $N$ electrons whose current density through the $z=0$ plane at radius $r$, azimuthal angle $\phi$, and time $t$ is $N q \rho(r, \phi) f(t)$, where $\int_{0}^{2 \pi} d \phi \int_{0}^{\infty} r d r \rho(r, \phi)=$ $\int_{-\infty}^{\infty} f(t) d t=1$. The current profile is $I(t)=N q f(t)$, while the form factor $F(\omega)=\int_{-\infty}^{\infty} f(t) \exp (i \omega t) d t$. The formation zone at wavelength $\lambda=2 \pi \sigma_{z}=2 \pi c \sigma_{t}$ approximates the bunch's formation zone [8,9], with formation length $\sigma_{z} \gamma^{2}$.

The longitudinal wake experienced by an on-axis electron that passes through the $z=0$ plane at time $\Delta t$ is [9]

$$
\begin{aligned}
W(z, \Delta t)= & (N / 2 \pi) \int_{-\infty}^{\infty} d \omega \exp [-i \omega(z / \beta c+\Delta t)] F(\omega) \\
& \times \int_{0}^{2 \pi} d \phi \int_{0}^{\infty} r d r \rho(r, \phi) \tilde{E}(r, z, \omega),
\end{aligned}
$$

where $\tilde{E}(r, z, \omega)$ is the single-electron field and $\beta \approx 1$ in the formation zone. The wake affecting any electron traveling parallel to the bunch may be calculated by considering its path to be the $z$ axis. For the single-electron field of Eq. (3), the wake downstream of the magnet is the sum of the Coulomb (LSC) wake of a bunch in uniform motion

$$
\begin{aligned}
W_{\text {Coul }}(z, \Delta t)= & N \int_{-\infty}^{\infty} F(\omega) e^{-i \omega \Delta t} d \omega \int_{0}^{\infty} r d r \bar{\rho}(r) \\
& \times\left[\left(\frac{-i q \omega}{2 \pi \varepsilon_{0} c^{2} \gamma^{2}}\right) K_{0}\left(\frac{|\omega| r}{c \gamma}\right)\right],
\end{aligned}
$$

and a coherent-radiation wake

$$
\begin{aligned}
W_{\mathrm{CR}}(z, \Delta t) & =N \int_{-\infty}^{\infty} F(\omega) e^{-i \omega \Delta t} d \omega \int_{0}^{\infty} r d r \bar{\rho}(r)\left(\frac{-q}{2 \pi \varepsilon_{0} c}\right) \frac{e^{-i k z}}{\sqrt{z^{2}+r^{2}}}\left(e^{i k \sqrt{z^{2}+r^{2}}}-e^{i k \sqrt{z^{2}+r^{2}} \cos \theta}\right) \\
& =\left(\frac{-N q}{\varepsilon_{0} c}\right) \int_{0}^{\infty} \frac{r d r \bar{\rho}(r)}{\sqrt{z^{2}+r^{2}}}\left[f\left(\Delta t-\frac{\sqrt{z^{2}+r^{2}}-z}{c}\right)-f\left(\Delta t-\frac{\sqrt{z^{2}+r^{2}} \cos \theta-z}{c}\right)\right] .
\end{aligned}
$$

Here, $\bar{\rho}(r) \equiv(2 \pi)^{-1} \int_{0}^{2 \pi} \rho(r, \phi) d \phi$ is the azimuthal average of $\rho(r, \phi)$.

For $\theta \leq \pi / 2$, Eq. (6) applies for a bunch of radius $r_{b}$ where $z \gg r_{b}$, while providing a reasonable approximation for $z \approx r_{b}$. For $\pi / 2<\theta \ll \pi-1 / \gamma$, Eq. (6) applies for $z \gg r_{b} / \sin \theta$ and approximates where $z \approx r_{b} / \sin \theta$.

Upstream of the magnet, the longitudinal field from an electron stopped at the entrance nearly equals its Coulomb field, while the field from an electron accelerated at the exit vanishes for $|z| \gg r$ when $\theta \leq \pi / 2$, and for $|z| \gg r / \sin \theta$ when $\pi / 2<\theta \ll \pi-1 / \gamma$. For $\theta \leq \pi / 2$, the coherentradiation wake upstream of the magnet nearly vanishes. For larger deflections, a coherent-radiation wake upstream of the magnet occurs for $|z| \lesssim r_{b} / \sin \theta$, where deflected electrons pass through the incident bunch.

We now consider a cylindrically symmetric bunch deflected through an angle $\theta \leq \pi / 2$, and let the path of the bunch center be the $z$ axis. For a uniform radial density distribution within radius $r_{b}\left[\bar{\rho}(r)=1 / \pi r_{b}^{2}\right.$ for $\left.r<r_{b}\right]$,

$$
\begin{aligned}
W_{\text {Coul }}(z, \Delta t)= & \frac{-N q}{2 \pi^{2} \varepsilon_{0} r_{b}^{2}} \int_{-\infty}^{\infty} F(\omega) e^{-i \omega \Delta t}\left(\frac{i}{\omega}\right) \\
& \times\left[1-\frac{|\omega| r_{b}}{c \gamma} K_{1}\left(\frac{|\omega| r_{b}}{c \gamma}\right)\right] d \omega
\end{aligned}
$$

and

$$
\begin{aligned}
W_{\mathrm{CR}}(z, \Delta t)= & \frac{-N q}{\pi \varepsilon_{0} r_{b}^{2}}\left[\int_{\Delta t-\left[\left(\sqrt{z^{2}+r_{b}^{2}}-z\right) / c\right]}^{\Delta t} f(t) d t\right. \\
& \left.-(\cos \theta)^{-1} \int_{\Delta t-\left[\left(\sqrt{z^{2}+r_{b}^{2}} \cos \theta-z\right) / c\right]}^{\Delta t-[(\cos \theta-z) / c]} f(t) d t\right] .
\end{aligned}
$$

For $r_{b} \ll \sigma_{z}$, a smooth distribution $f(t)$ is nearly constant within the integrals, giving 


$$
\begin{aligned}
W_{\mathrm{CR}}(z, \Delta t) \approx & \frac{-N q\left(\sqrt{z^{2}+r_{b}^{2}}-z\right) Z_{0}}{\pi r_{b}^{2}} \\
& \times\left[f(\Delta t)-f\left(\Delta t-\frac{z \cos \theta-z}{c}\right)\right],
\end{aligned}
$$

where $Z_{0}=\left(\varepsilon_{0} c\right)^{-1}=120 \pi$ ohms. For $r_{b} \geq \sigma_{z}$, Eq. (9) applies where $z \gg r_{b}^{2} / \sigma_{z}$.

In Eq. (9), the coherent-radiation wake is a resistive exitedge wake $[\propto N q f(\Delta t)=I(\Delta t)]$ summed with the entrance-edge wake, which moves ahead of the bunch. For $z \gg \max \left(r_{b}, r_{b}^{2} / \sigma_{z}\right)$ and $\theta \ll \pi / 2$, Eq. (9) becomes

$$
W_{\mathrm{CR}}(z, \Delta t) \approx \frac{-N q Z_{0}}{2 \pi z}\left[f(\Delta t)-f\left(\Delta t+\frac{z \theta^{2}}{2 c}\right)\right] .
$$

Equation (10) may also be obtained from the onedimensional CSR model of Ref. [2], which was extended in Ref. [3] to describe arbitrary bunch profiles within the formation zone. It is the sum of Eqs. (10) and (15) of Ref. [3], in the limit of zero bending radius.

\section{Weak magnet}

Consider a weak short magnet that deflects a bunch with uniform radial density through an angle $\theta \ll 1 / \gamma[11,14]$. Since $z \theta^{2} / 2 c \ll \sigma_{t}$ within the formation zone, the entrance-edge wake moves slightly ahead of the exitedge wake. The coherent-radiation wake results from their incomplete cancellation.

In Fig. 1 , the coherent-radiation wake for $\theta=0.1 / \gamma$ is calculated with Eq. (8) for a Gaussian longitudinal profile with energy of $511 \mathrm{MeV}$, charge of $200 \mathrm{pC}$, peak current of $50 \mathrm{~A}, \sigma_{z}=400 \mu \mathrm{m}$, and $r_{b}=0.1 \sigma_{z}=40 \mu \mathrm{m}$. These parameters represent a bunch from a photocathode [15]. The wake is calculated for a positive bunch charge, so that a negative wake corresponds to energy loss. For $z \gg$ $\max \left(r_{b}, r_{b}^{2} / \sigma_{z}\right)$, the wake agrees with Eq. (10).

Within the formation zone of a weak magnet, Eq. (9) is approximated by

$$
\begin{aligned}
W_{\mathrm{CR}}(z, \Delta t) \approx & \frac{N q\left(\sqrt{z^{2}+r_{b}^{2}}-z\right) Z_{0} \theta^{2}}{2 \pi r_{b}^{2}} \\
& \times\left[\frac{z}{c} f^{\prime}(\Delta t)+\frac{z^{2} \theta^{2}}{4 c^{2}} f^{\prime \prime}(\Delta t)\right] .
\end{aligned}
$$

For a typical smooth profile with $f^{\prime}(\Delta t) \sim f(\Delta t) / \sigma_{t}$ and $f^{\prime \prime}(\Delta t) \sim f(\Delta t) / \sigma_{t}^{2}$, Eq. (11) is approximated by $\left[N q\left(\sqrt{z^{2}+r_{b}^{2}}-z\right) Z_{0} \theta^{2} / 2 \pi r_{b}^{2}\right](z / c) f^{\prime}(\Delta t) \approx$ $\left[N q Z_{0} \theta^{2} / 4 \pi c\right] f^{\prime}(\Delta t)$. Since a weak magnet's coherentradiation wake is nearly independent of $z$ within the formation zone, and smaller than the LSC wake at the formation-zone boundary [9], it is smaller than the LSC wake everywhere. This is confirmed by Fig. 1.

The integrated coherent-radiation wake at a distance $z \gg \max \left(r_{b}, r_{b}^{2} / \sigma_{z}\right)$ is

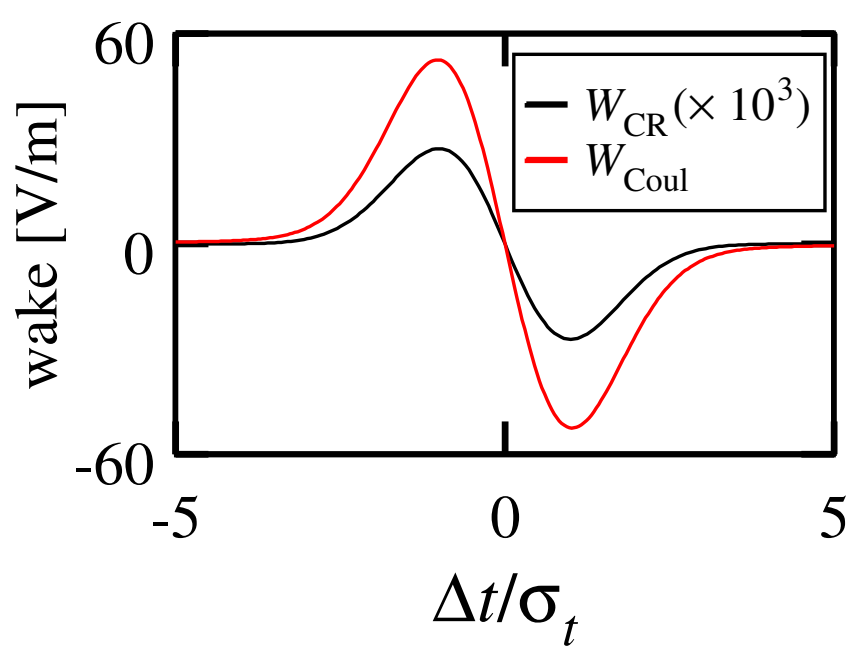

FIG. 1. (Color) Coherent-radiation wake $W_{\mathrm{CR}}$ and Coulomb (or LSC) wake $W_{\text {Coul }}$ for a weak magnet that deflects a bunch through the angle $\theta=0.1 / \gamma$. The bunch has a Gaussian longitudinal distribution, energy of $511 \mathrm{MeV}$, charge of $200 \mathrm{pC}$, rms length $\sigma_{z}=400 \mu \mathrm{m}$, and radius $r_{b}=0.1 \sigma_{z}$. The head of the bunch is on the left, while a negative wake corresponds to energy loss. The coherent-radiation wake at a distance of $100 \sigma_{z}$ downstream of the magnet is plotted. For $\sigma_{z} \leq z \leq 10^{5} \sigma_{z}$, the coherent-radiation wakes appear nearly identical.

$$
\begin{aligned}
\Delta V_{\mathrm{CR}}(z, \Delta t) & \approx \int_{0}^{z} W_{\mathrm{CR}}\left(z^{\prime}, \Delta t\right) d z^{\prime} \\
& \approx \frac{N q Z_{0} \theta^{2}}{4 \pi}\left[\frac{z}{c} f^{\prime}(\Delta t)+\frac{z^{2} \theta^{2}}{8 c^{2}} f^{\prime \prime}(\Delta t)\right]
\end{aligned}
$$

For a typical smooth profile, Eq. (12) is dominated by the term $\left(N q Z_{0} \theta^{2} z / 4 \pi c\right) f^{\prime}(\Delta t)$, while the bunch's energy loss results from the term $\left(N q Z_{0} \theta^{2} / 4 \pi\right)\left(z^{2} \theta^{2} / 8 c^{2}\right) f^{\prime \prime}(\Delta t)$.

\section{Strong magnet}

Consider a strong magnet that suddenly deflects a smooth bunch with uniform radial density through an angle with $1 / \gamma \ll \theta \ll \pi / 2$. Within the formation zone, the entrance-edge wake moves ahead of the bunch by a distance $\sigma_{z}$ where $z=\sigma_{z} /(1-\cos \theta) \approx 2 \sigma_{z} / \theta^{2}$. Figure 2 illustrates this behavior for $\theta=100 / \gamma$; the coherentradiation wakes shown agree with Eq. (10).

For $\max \left(r_{b}, r_{b}^{2} / \sigma_{z}\right) \ll z \ll \sigma_{z} / \theta^{2}$, Eqs. (11) and (12) apply. This region's contribution to the integrated wake, estimated by setting $z=\sigma_{z} / \theta^{2}$ in Eq. (12), is dominated by $\left(N q Z_{0} / 4 \pi\right) \sigma_{t} f^{\prime}(\Delta t)$. This region is shown in Fig. 2 for $z=10 \sigma_{z}=4 \mathrm{~mm}$.

When $z=2 \sigma_{z} / \theta^{2}$, the entrance-edge wake has moved ahead of the bunch by a distance $\sigma_{z}$, shown in Fig. 2 for $z=200 \sigma_{z}=80 \mathrm{~mm}$. When $z=12 \sigma_{z} / \theta^{2}$, the entranceedge wake has overtaken the bunch by a distance $6 \sigma_{z}$, as seen for $z=1200 \sigma_{z}=0.48 \mathrm{~m}$.

For $z \gg \sigma_{z} / \theta^{2}$, the entrance-edge wake does not affect the bunch. In the formation zone, the bunch experiences 

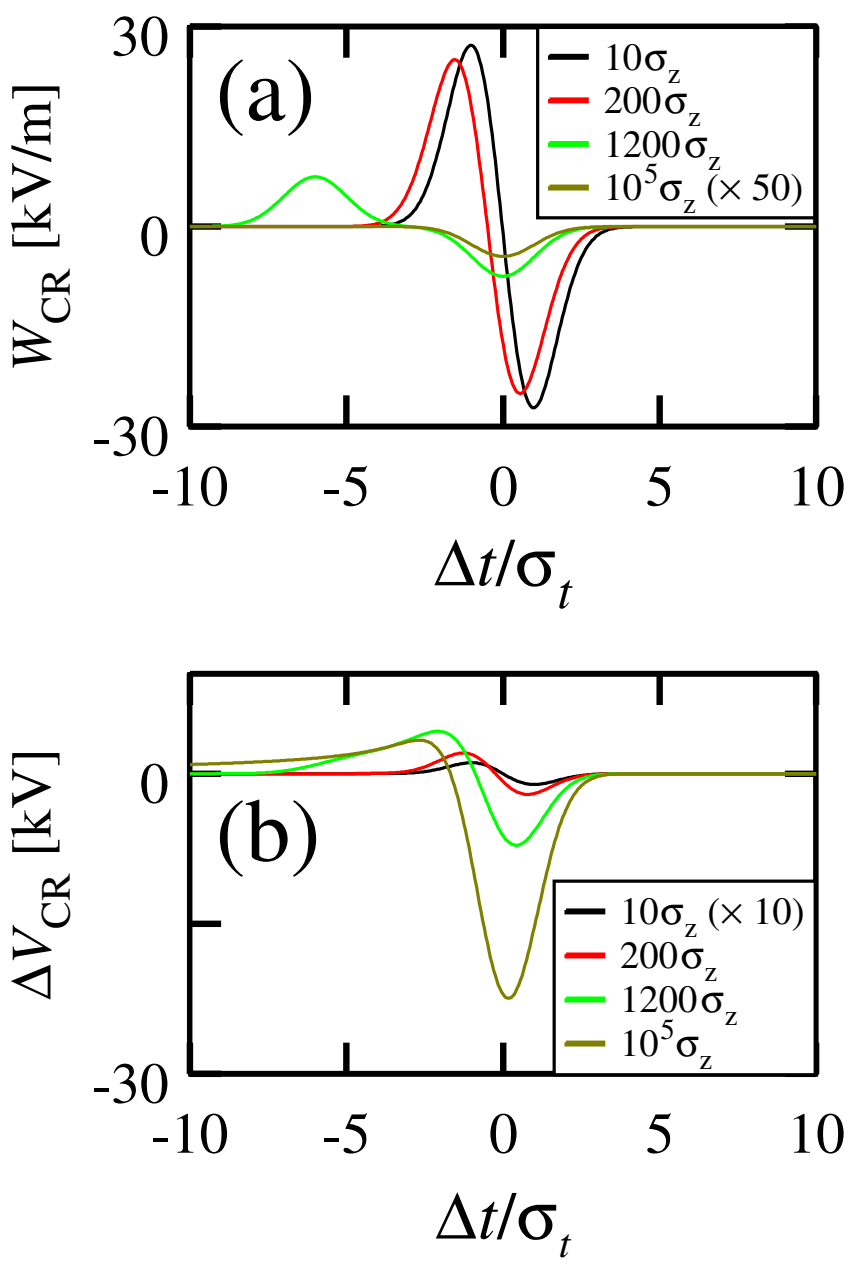

FIG. 2. (Color) Coherent-radiation wakes for a strong magnet that deflects a bunch through the angle $\theta=100 / \gamma$. The 511$\mathrm{MeV}$ 200-pC Gaussian bunch has $\sigma_{z}=400 \mu \mathrm{m}$ and $r_{b}=$ $0.1 \sigma_{z}$. (a) Coherent-radiation wake at four distances downstream of the magnet. (b) Integrated coherent-radiation wake at the same four distances.

the resistive exit-edge wake [9]

$$
\begin{aligned}
W_{\mathrm{CR}}(z, \Delta t) & =\frac{-N q}{\pi \varepsilon_{0} r_{b}^{2}} \int_{\Delta t-\left[\left(\sqrt{z^{2}+r_{b}^{2}}-z\right) / c\right]}^{\Delta t} f(t) d t \\
& \approx \frac{-N q f(\Delta t)}{\pi \varepsilon_{0} c r_{b}^{2}}\left(\sqrt{z^{2}+r_{b}^{2}}-z\right) \\
& \approx \frac{-N q f(\Delta t) Z_{0}}{2 \pi z} .
\end{aligned}
$$

The approximate expressions in Eq. (13) apply for $r_{b} \ll$ $\sigma_{z} / \theta$. For drift length $L_{d} \gg \sigma_{z} / \theta^{2}$, integrating Eq. (13) over $\sigma_{z} / \theta^{2}<z<\min \left(L_{d}, \sigma_{z} \gamma^{2}\right)$ estimates the integrated coherent-radiation wake of a strong short magnet

$$
\Delta V_{\mathrm{CR}}\left(z=L_{d}, \Delta t\right) \approx \frac{-N q f(\Delta t) Z_{0}}{2 \pi} \ln \left(\frac{\min \left(L_{d}, \sigma_{z} \gamma^{2}\right)}{\sigma_{z} / \theta^{2}}\right) .
$$

The integrated coherent-radiation wake is approximated by resistive impedance $\left(Z_{0} / 2 \pi\right) \ln \left[\min \left(L_{d}, \sigma_{z} \gamma^{2}\right) /\left(\sigma_{z} / \theta^{2}\right)\right]$; the impedance at wavelength $\lambda$ is estimated by setting $\sigma_{z}=\lambda / 2 \pi$. For $L_{d}>\sigma_{z} \gamma^{2}$, the impedance is $\sim\left(Z_{0} / \pi\right) \times$ $\ln (\gamma \theta)$. The small coherent-radiation wake for $z \gtrsim \sigma_{z} \gamma^{2}$ can be computed from Eq. (A4).

\section{E. Applicability of formulas}

For deflection over a distance $d$ that is small compared to the formation length of steady-state CSR, our model includes the bunch's transverse dimensions in formulas that apply for $d \ll z \ll \sigma_{z} \gamma^{2}$ and $z \sin \theta \ll \sigma_{z} \gamma$, where the observer is in the formation zone of the entrance edge radiation. Our formulas give the wake affecting the bunch for $d \ll z \ll \sigma_{z} \gamma^{2}$, but for $z \sin \theta>\sigma_{z} \gamma$, our formulas do not model the entrance-edge wake of a strong magnet after it has moved far ahead of the bunch. For $d \ll$ $\max \left(r_{b}, r_{b}^{2} / \sigma_{z}\right)$, which occurs when modeling transition radiation, integrating our wake formulas over $(0, z)$ gives the integrated wake for $z \gg \max \left(r_{b}, r_{b}^{2} / \sigma_{z}\right)$ [9].

According to Eqs. (8)-(10), the one-dimensional CSR model [2,3] describes a short magnet with $\theta \ll \pi / 2$ for $z \gg \max \left(r_{b}, r_{b}^{2} / \sigma_{z}\right)$. The wake downstream of a long magnet with radius of curvature $\rho_{0}$ obeys the onedimensional model when the steady-state CSR formation length $\sim \rho_{0}^{2 / 3}\left(2 \pi \sigma_{z}\right)^{1 / 3}$ exceeds $\max \left(r_{b}, r_{b}^{2} / \sigma_{z}\right)$ [9]. Thus, the one-dimensional CSR model applies downstream of a magnet of length $d$ when

$$
\min \left[d, \rho_{0}^{2 / 3}\left(2 \pi \sigma_{z}\right)^{1 / 3}\right] \gg \max \left(r_{b}, r_{b}^{2} / \sigma_{z}\right) .
$$

In this case, the one-dimensional ultrarelativistic model [3] used by the ELEGANT code [4] gives the wake affecting the bunch, making an approximation error for $z \gtrsim \sigma_{z} \gamma^{2}$ that is smaller than the LSC wake.

In a vacuum chamber of height $h$, where the wake is shielded for $z \gg \max \left(h, h^{2} / 2 \pi \sigma_{z}\right)$ [9], our formulas do not apply for $z \gtrsim \max \left(h, h^{2} / 2 \pi \sigma_{z}\right)$.

\section{COHERENT TRANSITION AND DIFFRACTION RADIATION}

\section{A. Transition radiation}

Consider forward transition radiation (FTR) from an ultrarelativistic electron that exits a conductor at angle $\theta_{\text {target }} \leq \pi / 2$ with respect to the surface. The radiation is the sum of the fields of a suddenly accelerated electron and a positive image charge that is suddenly accelerated at an angle of $2 \theta_{\text {target }}$ with respect to the electron. The image charge field equals that of a suddenly stopped electron summed with the Coulomb field of an image charge in uniform motion. Thus, FTR is the radiation of an electron deflected through the angle $2 \theta_{\text {target }}$ summed with the Coulomb field of an image charge.

The backward transition radiation (BTR) of an electron that enters a conductor at angle $\theta_{\text {target }}$ is the radiation of a 
suddenly stopped electron summed with the radiation of a suddenly stopped image charge, equal to the radiation from an electron deflected through the angle $2 \theta_{\text {target }}$ summed with the Coulomb field of an image charge.

In the far field, where the Coulomb field vanishes, the transition radiation equals the radiation of a suddenly deflected electron, given by the sum of two radially polarized symmetrical cones with opening angles of $1 / \gamma$ [11-13]. For an inclined target with $1 / \gamma \ll \theta_{\text {target }}<\pi / 2$, the sum consists of two slightly asymmetric cones [16]. In the formation zone, the coherent-radiation field is dominated by the radially polarized symmetrical field of a suddenly stopped charge [Eq. (A6)] with opening angle of $0.86(\lambda / R)^{1 / 2}$; the sum of the fields from a charge and its image causes cylindrical asymmetry for an inclined target $[17,18]$.

Since a bunch's FTR wake is the wake of suddenly deflected electrons summed with the Coulomb wake of an image bunch, the longitudinal coherent-radiation wake obeys Eq. (4) with the single-electron field $\tilde{E}=\tilde{E}_{\text {exit }}+$ $\tilde{E}_{\text {ent }}-\tilde{\boldsymbol{E}}_{\text {Coul }} \cdot \hat{z}$. Here, $\tilde{\boldsymbol{E}}_{\text {Coul }} \cdot \hat{z}$ is the longitudinal component of the Coulomb field given by Eq. (A10) for an electron traveling in the direction of the image bunch. This field cancels $\tilde{E}_{\text {ent }}$, so that the FTR wake is the wake of suddenly accelerated electrons [9]

$$
\begin{aligned}
W_{\mathrm{FTR}}(z, \Delta t)= & \left(\frac{-N q}{\varepsilon_{0} c}\right) \int_{0}^{\infty} \frac{r d r \bar{\rho}(r)}{\sqrt{z^{2}+r^{2}}} \\
& \times f\left(\Delta t-\frac{\sqrt{z^{2}+r^{2}}-z}{c}\right) .
\end{aligned}
$$

The BTR wake occurs upstream of the conductor where $z<0$. The coherent-radiation wake from a suddenly stopped image bunch is observed at angle $\theta=$ $\pi-2 \theta_{\text {target }}$. Since the field of an image charge is the opposite of that given by Eq. (2),

$$
\begin{aligned}
W_{\mathrm{BTR}}(z, \Delta t) \approx & \left(\frac{N q}{\varepsilon_{0} c}\right) \int_{0}^{\infty} \frac{r d r \bar{\rho}(r)}{\sqrt{z^{2}+r^{2}}} \\
& \times f\left(\Delta t+\frac{\sqrt{z^{2}+r^{2}} \cos \left(2 \theta_{\text {target }}\right)+z}{c}\right) .
\end{aligned}
$$

Equation (16) applies when $\tilde{E}_{\text {ent }}$ is canceled by $\tilde{\boldsymbol{E}}_{\text {Coul }} \cdot \hat{z}$, while Eq. (17) applies when the field of an image charge is the opposite of that given by Eq. (2). In both cases this requires $\theta_{\text {target }} \gg 1 / \gamma$ and $|z| \sin \theta_{\text {target }} \gg r$. For bunch radius $r_{b}$, Eqs. (16) and (17) apply for $|z| \gg r_{b} / \sin \theta_{\text {target }}$ (where the bunch slice does not intersect the target) and provide an approximation for $|z| \approx r_{b} / \sin \theta_{\text {target }}$. For the common target geometries with $\theta_{\text {target }} \geq \pi / 4$, Eqs. (16) and (17) apply for $|z| \gg r_{b}$ and provide an approximation for $|z| \approx r_{b}$; the BTR wake primarily affects the bunch where $|z|<\sigma_{z}$.
The longitudinal wake is the coherent-radiation FTR or BTR wake summed with the LSC wake. To determine the wake affecting any electron traveling parallel to the bunch, one may let its path be the $z$ axis and calculate $\bar{\rho}(r) \equiv$ $(2 \pi)^{-1} \int d \phi \rho(r, \phi)$ for this axis.

\section{B. Circular iris and disk}

Consider a cylindrically symmetric bunch that passes through the center of a hole of radius $a \ll \sigma_{z} \gamma$ in a perpendicular conducting plane, producing coherent diffraction radiation (CDR) [16]. At radii where $a<r \ll$ $\sigma_{z} \gamma$, the Coulomb field is radially polarized and inversely proportional to radius, nearly equal to the field of a hollow bunch with radius $a$. Thus, CDR is modeled by the coherent transition radiation from a hollow bunch. The formation-zone wake of forward diffraction radiation (FDR) is given by Eq. (16) with $\bar{\rho}(r)=\delta(r-a) / 2 \pi a$

$$
W_{\text {iris }}(z, \Delta t)=\left(\frac{-N q}{2 \pi \varepsilon_{0} c \sqrt{z^{2}+a^{2}}}\right) f\left(\Delta t-\frac{\sqrt{z^{2}+a^{2}}-z}{c}\right) .
$$

In Appendix B, we obtain Eq. (18) from a Kirchhoff diffraction integral over the target.

For $\max \left(a, a^{2} / \sigma_{z}\right) \ll z \ll \sigma_{z} \gamma^{2}$, this wake gives resistance per unit length of $Z_{0} / 2 \pi z$. For an iris with $a>\sigma_{z} \gamma$ that does not intercept the transverse Coulomb field, Eq. (18) gives a negligible wake for $z \ll \sigma_{z} \gamma^{2}$. Thus, Eq. (18) and subsequent CDR formulas apply for target radii exceeding $\sigma_{z} \gamma$, but the wakes only apply for $z \ll$ $\sigma_{z} \gamma^{2}$.

For a tilted target, the transition radiation of a hollow round bunch models the diffraction radiation of an elliptical iris whose projection onto the normal plane is a circle. The FDR wake for a normal-incidence geometry applies to a tilted target whose normal projection has the same geometry. For the common case $\theta_{\text {target }} \geq \pi / 4$, where Eqs. (16) and (17) apply for $|z| \gg r_{b}$ and approximate for $|z| \approx r_{b}$, our CDR formulas apply for $|z| \gg a$ while providing an approximation for $|z| \approx a$.

By superposition, the FDR wake from a disk with inner radius $a$ and outer radius $b$ is the difference between the wakes of irises with radii $a$ and $b$ :

$$
\begin{aligned}
W_{\mathrm{FDR}}(z, \Delta t)= & \left(\frac{-N q}{2 \pi \varepsilon_{0} c}\right)\left[\frac{f\left[\Delta t-\left(\sqrt{z^{2}+a^{2}}-z\right) / c\right]}{\sqrt{z^{2}+a^{2}}}\right. \\
& \left.-\frac{f\left[\Delta t-\left(\sqrt{z^{2}+b^{2}}-z\right) / c\right]}{\sqrt{z^{2}+b^{2}}}\right] .
\end{aligned}
$$

In the formation zone, this wake gives resistance per unit length of $Z_{0} / 2 \pi z$ for $\max \left(a, a^{2} / \sigma_{z}\right) \ll z \ll$ $\max \left(b, b^{2} / \sigma_{z}\right)$. The integrated wake for $\sigma_{z}<a \ll b \ll$ $\sigma_{z} \gamma$ is approximated by resistance $\left(Z_{0} / \pi\right) \ln (b / a)$, in 
agreement with previous calculations $[5,7,19]$. In a vacuum chamber whose height exceeds $2 b$, the FDR wake decays before significant shielding occurs, so that Eq. (19) applies.

Similarly, the backward diffraction-radiation (BDR) wake of a disk is
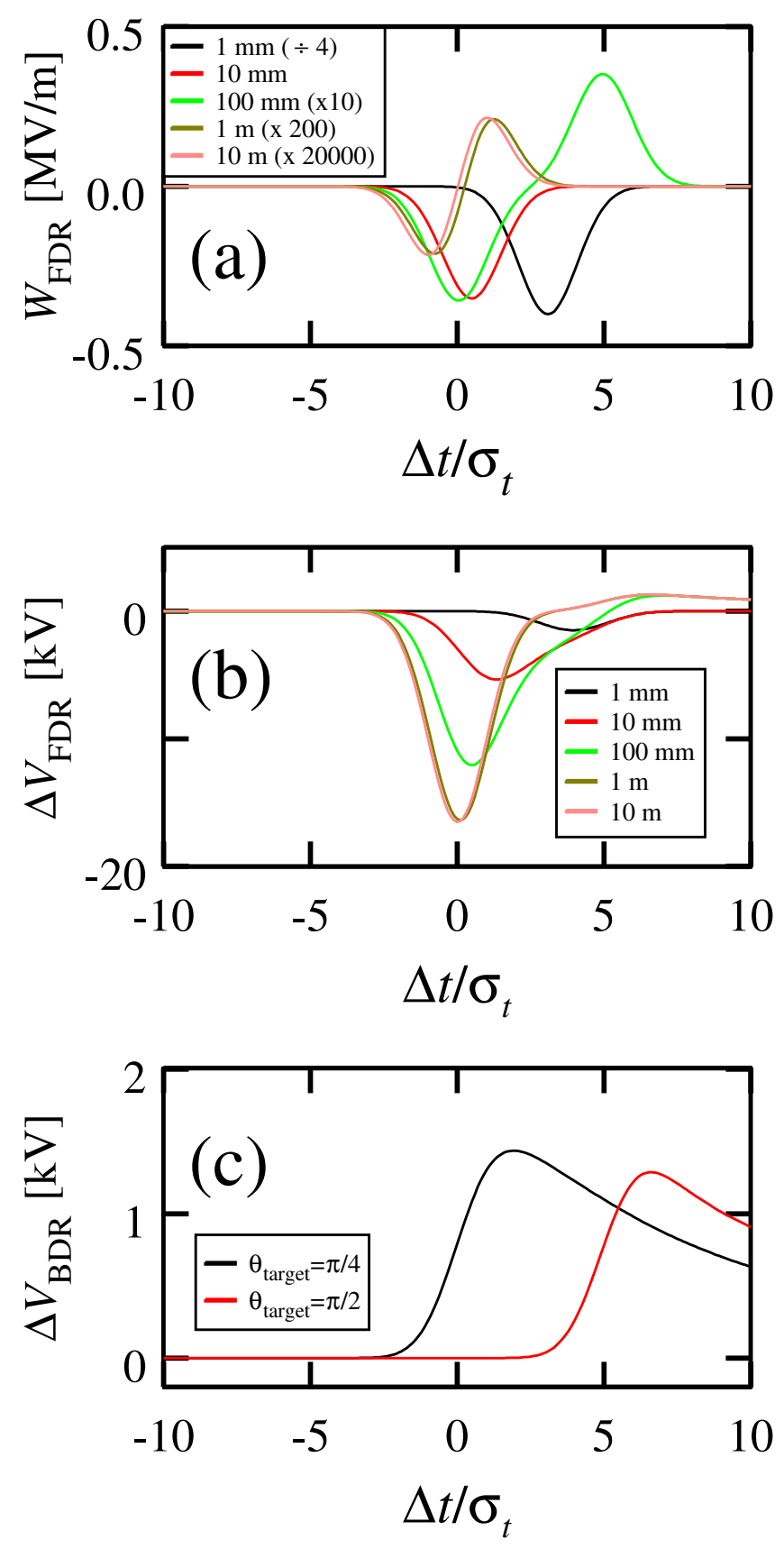

FIG. 3. (Color) Coherent diffraction-radiation (CDR) wakes of a disk target with inner radius of $2 \mathrm{~mm}$ and outer radius of $20 \mathrm{~mm}$, for a 511-MeV 200-pC Gaussian bunch with $\sigma_{z}=400 \mu \mathrm{m}$ and $r_{b}=0.1 \sigma_{z}$. (a) Forward diffraction-radiation (FDR) wake at five distances downstream of the target. (b) Integrated FDR wake at the same five distances. (c) Integrated wakes of backward diffraction radiation (BDR) for two target orientations.

$$
\begin{aligned}
W_{\mathrm{BDR}}(z, \Delta t)= & \left(\frac{N q}{2 \pi \varepsilon_{0} c}\right) \\
& \times\left[\frac{f\left\{\Delta t+\left[\sqrt{z^{2}+a^{2}} \cos \left(2 \theta_{\text {target }}\right)+z\right] / c\right\}}{\sqrt{z^{2}+a^{2}}}\right. \\
& \left.-\frac{f\left\{\Delta t+\left[\sqrt{z^{2}+b^{2}} \cos \left(2 \theta_{\text {target }}\right)+z\right] / c\right\}}{\sqrt{z^{2}+b^{2}}}\right] .
\end{aligned}
$$

For a target with $\theta_{\text {target }} \geq \pi / 4$ and $a \gg \sigma_{z}$, the BDR wake has little effect on the bunch.

Figure 3 shows the FDR wake for a disk with inner radius of $2 \mathrm{~mm}$ and outer radius of $20 \mathrm{~mm}$. The integrated wake agrees with resistive impedance of $\left(Z_{0} / \pi\right) \ln (b / a)$. The small integrated wakes of BDR are shown for the most common target geometries.

\section{Arbitrary target shape}

A disk with inner radius $r$ and outer radius $r+d r$ contributes the coherent wake

$$
d W_{\mathrm{CR}}(z, \Delta t)=\left(\frac{N q}{2 \pi \varepsilon_{0} c}\right) d\left[\frac{f\left[\Delta t-\left(\sqrt{z^{2}+r^{2}}-z\right) / c\right]}{\sqrt{z^{2}+r^{2}}}\right] .
$$

Since Eqs. (16) and (B1) indicate that the contribution of a target region is independent of azimuth, the contribution from part of a disk that subtends the azimuthal angle $\Delta \phi(r)$ is obtained by multiplying by $\Delta \phi(r) / 2 \pi$. When a target intercepts a cylindrically symmetric bunch, Eq. (21) must be multiplied by the portion of beam within radius $r$, $P(r) \equiv 2 \pi \int_{0}^{r} r^{\prime} d r^{\prime} \bar{\rho}\left(r^{\prime}\right)$. Integrating by parts gives

$$
\begin{aligned}
W_{\mathrm{CR}}(z, \Delta t)= & \left(\frac{N q}{2 \pi \varepsilon_{0} c}\right) \int_{0}^{\infty} P(r) \frac{\Delta \phi(r)}{2 \pi} \\
& \times \frac{d}{d r}\left[\frac{f\left[\Delta t-\left(\sqrt{z^{2}+r^{2}}-z\right) / c\right]}{\sqrt{z^{2}+r^{2}}}\right] d r \\
= & \left(\frac{-N q}{4 \pi^{2} \varepsilon_{0} c}\right) \int_{0}^{\infty}\left[\frac{f\left[\Delta t-\left(\sqrt{z^{2}+r^{2}}-z\right) / c\right]}{\sqrt{z^{2}+r^{2}}}\right] \\
& \times \frac{d}{d r}[P(r) \Delta \phi(r)] d r .
\end{aligned}
$$

For $\sigma_{z} \ll r_{b} \ll a$ and $\Delta \phi(r)=2 \pi$ for $r<a$, Eq. (22) reproduces Eq. (34) of Ref. [6].

For a target that does not intercept the bunch, setting $P(r)=1$ gives the FDR wake

$$
\begin{aligned}
W_{\mathrm{FDR}}(z, \Delta t)= & \left(\frac{-N q}{4 \pi^{2} \varepsilon_{0} c}\right) \int_{0}^{\infty} d r \\
& \times\left[\frac{f\left[\Delta t-\left(\sqrt{z^{2}+r^{2}}-z\right) / c\right]}{\sqrt{z^{2}+r^{2}}}\right] \frac{d}{d r} \Delta \phi(r) .
\end{aligned}
$$


Similarly, the BDR wake is

$$
W_{\mathrm{BDR}}(z, \Delta t)=\left(\frac{N q}{4 \pi^{2} \varepsilon_{0} c}\right) \int_{0}^{\infty} d r\left[\frac{f\left\{\Delta t+\left[\sqrt{z^{2}+r^{2}} \cos \left(2 \theta_{\text {target }}\right)+z\right] / c\right\}}{\sqrt{z^{2}+r^{2}}}\right] \frac{d}{d r} \Delta \phi(r) .
$$
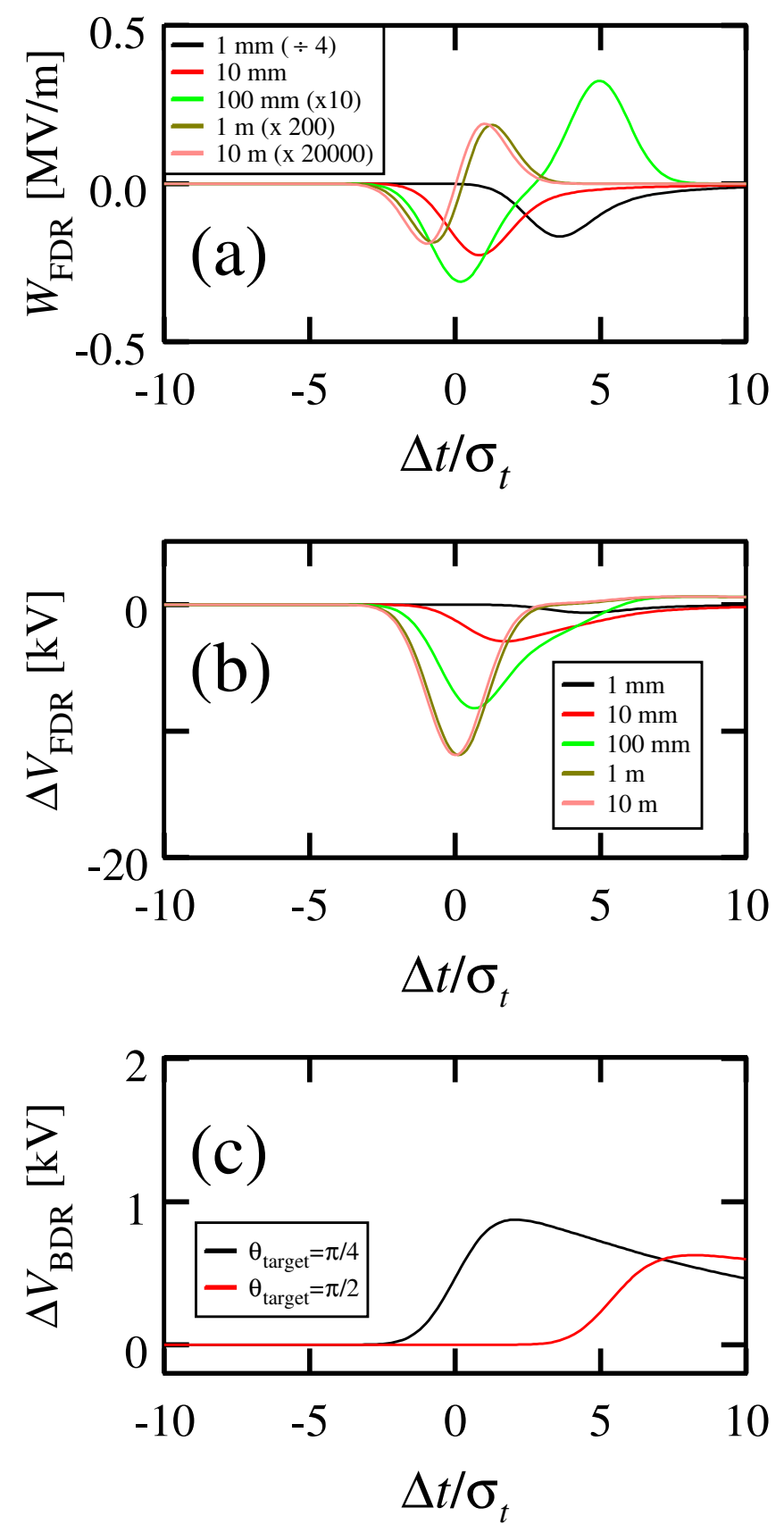

FIG. 4. (Color) Coherent diffraction-radiation (CDR) wakes of a slit between two blades with offsets of $2 \mathrm{~mm}$ and outer radii of $20 \mathrm{~mm}$, for a 511-MeV 200-pC Gaussian bunch with $\sigma_{z}=$ $400 \mu \mathrm{m}$ and $r_{b}=0.1 \sigma_{z}$. (a) Forward diffraction-radiation (FDR) wake at five distances downstream of the target. (b) Integrated FDR wake at the same five distances. (c) Integrated wakes of backward diffraction radiation (BDR) for two target orientations.
Here, the $z$ axis is the path of the bunch. The wake of a bunch that passes through a target off center is given by calculating $\Delta \phi(r)$ with respect to the off-center axis.

The CDR wake of a disk is given by $\Delta \phi(r)=2 \pi H(r-$ a) $H(b-r)$, where $H(x)$ is the Heaviside function and $d / d r[\Delta \phi(r)]=2 \pi[\delta(r-a)-\delta(r-b)]$. For a blade with offset $a$ and outer radius $b, \Delta \phi(r)=$ $2 \cos ^{-1}(a / r) H(r-a) H(b-r) \quad$ and $\quad d / d r[\Delta \phi(r)]=$ $\left(2 a / r \sqrt{r^{2}-a^{2}}\right) H(r-a) H(b-r)-2 \cos ^{-1}(a / b) \delta(r-b)$. For a slit with half-width $a$ formed from a pair of identical blades, the CDR wake is twice as large. Figure 4 shows the CDR wakes for a pair of blades with $a=2 \mathrm{~mm}$ and $b=20 \mathrm{~mm}$.

\section{Upstream edge radiation}

A target downstream of a bending magnet extracts some of the magnet's synchrotron radiation. For infrared wavelengths, where the synchrotron radiation is dominated by edge radiation [20,21], the transverse coherent-radiation field at distance $L$ from the magnet is intercepted by a target with outer radius $\gtrsim 0.86\left(2 \pi \sigma_{z} L\right)^{1 / 2}$.

For a normal-incidence target with $\max \left[\sigma_{z}\right.$, $\left.\rho_{0}^{2 / 3}\left(2 \pi \sigma_{z}\right)^{1 / 3}\right] \ll L \ll \sigma_{z} \gamma^{2}$ and $\max \left(r_{b}, r_{b}^{2} / \sigma_{z}\right) \ll L$, the coherent radiation in the target plane is formationzone edge radiation that is unaffected by the bunch radius [9]. For this case, we calculate the wake downstream of the target from edge radiation that passes through the "opening" in the target plane that is not occupied by the target. For an electron that passes the target at $t=0$, Eq. (1) gives the longitudinal coherent-radiation field in the opening at radius $r$ :

$$
\Delta \tilde{E}_{\mathrm{open}}=\frac{-q e^{i k\left(\sqrt{L^{2}+r^{2}}-L\right)}}{2 \pi \varepsilon_{0} c \sqrt{L^{2}+r^{2}}}
$$

A Kirchhoff diffraction integral over the area $A$ of the opening gives the on-axis edge-radiation field at distance $z$ downstream of the target

$$
\Delta \tilde{E}(z, \omega)=\frac{-i k}{2 \pi} \int_{\text {open }} d A \Delta \tilde{E}_{\text {open }} \frac{e^{i k \sqrt{z^{2}+r^{2}}}}{\sqrt{z^{2}+r^{2}}}
$$

Letting $d A=r d r \Delta \phi_{\text {open }}(r)$, where $\Delta \phi_{\text {open }}(r)$ is the angular extent of the opening, the formation-zone edgeradiation wake $(N / 2 \pi) \int d \omega \exp (-i k z-i \omega \Delta t) F(\omega) \times$ $\Delta \tilde{E}(z, \omega)$ is 


$$
\begin{aligned}
\Delta W_{\text {down }}(z, \Delta t) & =\frac{i N q}{8 \pi^{3} \varepsilon_{0} c^{2}} \int_{0}^{\infty} r d r \frac{\Delta \phi_{\text {open }}(r)}{\sqrt{L^{2}+r^{2}} \sqrt{z^{2}+r^{2}}} \int_{-\infty}^{\infty} \omega d \omega F(\omega) e^{-i \omega \Delta t} e^{i k\left(\sqrt{L^{2}+r^{2}}-L\right)} e^{i k\left(\sqrt{z^{2}+r^{2}}-z\right)} \\
& =\frac{-N q}{4 \pi^{2} \varepsilon_{0} c^{2}} \int_{0}^{\infty} r d r \frac{\Delta \phi_{\text {open }}(r)}{\sqrt{L^{2}+r^{2}} \sqrt{z^{2}+r^{2}}} f^{\prime}\left(\Delta t-\frac{\sqrt{L^{2}+r^{2}}-L}{c}-\frac{\sqrt{z^{2}+r^{2}}-z}{c}\right) .
\end{aligned}
$$

Noting that

$$
\frac{d}{d r} f\left(\Delta t-\frac{\sqrt{L^{2}+r^{2}}-L}{c}-\frac{\sqrt{z^{2}+r^{2}}-z}{c}\right)=\frac{-r}{c}\left(\frac{\sqrt{z^{2}+r^{2}}+\sqrt{L^{2}+r^{2}}}{\sqrt{L^{2}+r^{2}} \sqrt{z^{2}+r^{2}}}\right) f^{\prime}\left(\Delta t-\frac{\sqrt{L^{2}+r^{2}}-L}{c}-\frac{\sqrt{z^{2}+r^{2}}-z}{c}\right),
$$

we integrate Eq. (27) by parts to obtain

$$
\begin{aligned}
\Delta W_{\text {down }}(z, \Delta t) & =\frac{-N q f(\Delta t)}{2 \pi \varepsilon_{0} c(L+z)}-\frac{N q}{4 \pi^{2} \varepsilon_{0} c} \int_{0}^{\infty} d r \frac{d}{d r}\left(\frac{\Delta \phi_{\text {open }}(r)}{\sqrt{L^{2}+r^{2}}+\sqrt{z^{2}+r^{2}}}\right) f\left(\Delta t-\frac{\sqrt{L^{2}+r^{2}}-L}{c}-\frac{\sqrt{z^{2}+r^{2}}-z}{c}\right) \\
& \approx \frac{-N q f(\Delta t)}{2 \pi \varepsilon_{0} c(L+z)}+\frac{N q}{4 \pi^{2} \varepsilon_{0} c} \int_{0}^{\infty} d r \frac{\frac{d}{d r} \Delta \phi(r)}{\sqrt{L^{2}+r^{2}}+\sqrt{z^{2}+r^{2}}} f\left(\Delta t-\frac{\sqrt{L^{2}+r^{2}}-L}{c}-\frac{\sqrt{z^{2}+r^{2}}-z}{c}\right), \quad(29)
\end{aligned}
$$

where $\Delta \phi(r)=2 \pi-\Delta \phi_{\text {open }}(r)$ is the angular extent of the target at radius $r$. The approximation in Eq. (29) applies for $\sigma_{z} \ll L$. Adding Eqs. (23) and (29) gives the coherent-radiation wake downstream of the target

$$
W_{\text {down }}(z, \Delta t)=\frac{-N q f(\Delta t)}{2 \pi \varepsilon_{0} c(L+z)}-\frac{N q}{4 \pi^{2} \varepsilon_{0} c} \int_{0}^{\infty} d r\left[\frac{f\left(\Delta t-\frac{\sqrt{z^{2}+r^{2}}-z}{c}\right)}{\sqrt{z^{2}+r^{2}}}-\frac{f\left(\Delta t-\frac{\sqrt{L^{2}+r^{2}}-L}{c}-\frac{\sqrt{z^{2}+r^{2}}-z}{c}\right)}{\sqrt{L^{2}+r^{2}}+\sqrt{z^{2}+r^{2}}}\right] \frac{d}{d r} \Delta \phi(r) .
$$

The first term of Eq. (30) is the coherent edge-radiation wake when there is no target. Subtracting this term gives the downstream wake perturbation from the target

$$
W_{\text {pert }}(z, \Delta t)=\frac{-N q}{4 \pi^{2} \varepsilon_{0} c} \int_{0}^{\infty} d r\left[\frac{f\left(\Delta t-\frac{\sqrt{z^{2}+r^{2}}-z}{c}\right)}{\sqrt{z^{2}+r^{2}}}-\frac{f\left(\Delta t-\frac{\sqrt{L^{2}+r^{2}}-L}{c}-\frac{\sqrt{z^{2}+r^{2}}-z}{c}\right)}{\sqrt{L^{2}+r^{2}}+\sqrt{z^{2}+r^{2}}}\right] \frac{d}{d r} \Delta \phi(r) .
$$

The first term in Eq. (31) is FDR, while the second term is from diffracted edge radiation.

Upstream of a normal-incidence target, a Kirchhoff diffraction integral gives the longitudinal wake from reflected edge radiation

$$
\Delta W_{\text {up }}(z, \Delta t)=\frac{-N q}{4 \pi^{2} \varepsilon_{0} c} \int_{0}^{\infty} d r \frac{\frac{d}{d r} \Delta \phi(r)}{\sqrt{L^{2}+r^{2}}+\sqrt{z^{2}+r^{2}}} f\left(\Delta t-\frac{\sqrt{L^{2}+r^{2}}-L}{c}-\frac{\sqrt{z^{2}+r^{2}}-z}{c}\right) .
$$

For a tilted target, we approximate the modification of the upstream wake by that of BDR

$$
\Delta W_{\mathrm{up}}(z, \Delta t) \approx \frac{-N q}{4 \pi^{2} \varepsilon_{0} c} \int_{0}^{\infty} d r \frac{\frac{d}{d r} \Delta \phi(r)}{\sqrt{L^{2}+r^{2}}+\sqrt{z^{2}+r^{2}}} f\left(\Delta t-\frac{\sqrt{L^{2}+r^{2}}-L}{c}+\frac{\sqrt{z^{2}+r^{2}} \cos \left(2 \theta_{\text {target }}\right)+z}{c}\right) .
$$

The wake perturbation upstream of the target is the sum of Eq. (33) and the BDR wake

$$
W_{\text {pert }}(z, \Delta t) \approx \frac{N q}{4 \pi^{2} \varepsilon_{0} c} \int_{0}^{\infty} d r\left[\frac{f\left(\Delta t+\frac{\sqrt{z^{2}+r^{2}} \cos \left(2 \theta_{\text {target }}\right)+z}{c}\right)}{\sqrt{z^{2}+r^{2}}}-\frac{f\left(\Delta t-\frac{\sqrt{L^{2}+r^{2}}-L}{c}+\frac{\sqrt{z^{2}+r^{2}} \cos \left(2 \theta_{\text {target }}\right)+z}{c}\right)}{\sqrt{L^{2}+r^{2}}+\sqrt{z^{2}+r^{2}}}\right] \frac{d}{d r} \Delta \phi(r) .
$$

Figure 5 displays integrated wakes $10 \mathrm{~m}$ downstream of a disk target that is located $1 \mathrm{~m}$ downstream of a bending magnet. For a target with inner radius of $2 \mathrm{~mm}$ and outer radius of $20 \mathrm{~mm}$, wakes are shown for 200-pC 511-MeV Gaussian bunches with rms bunch lengths of 400, 100, and $50 \mu \mathrm{m}$. We show the integrated edge-radiation wake when there is no target, the perturbation from the target, and the total coherent-radiation wake given by their sum. For comparison, we show the integrated FDR wake.

For the longest bunch length of $400 \mu \mathrm{m}$, the target's outer radius is less than half of $0.86\left(2 \pi \sigma_{z} L\right)^{1 / 2}=43 \mathrm{~mm}$. In this case, the diffracted radiation is approximated by 


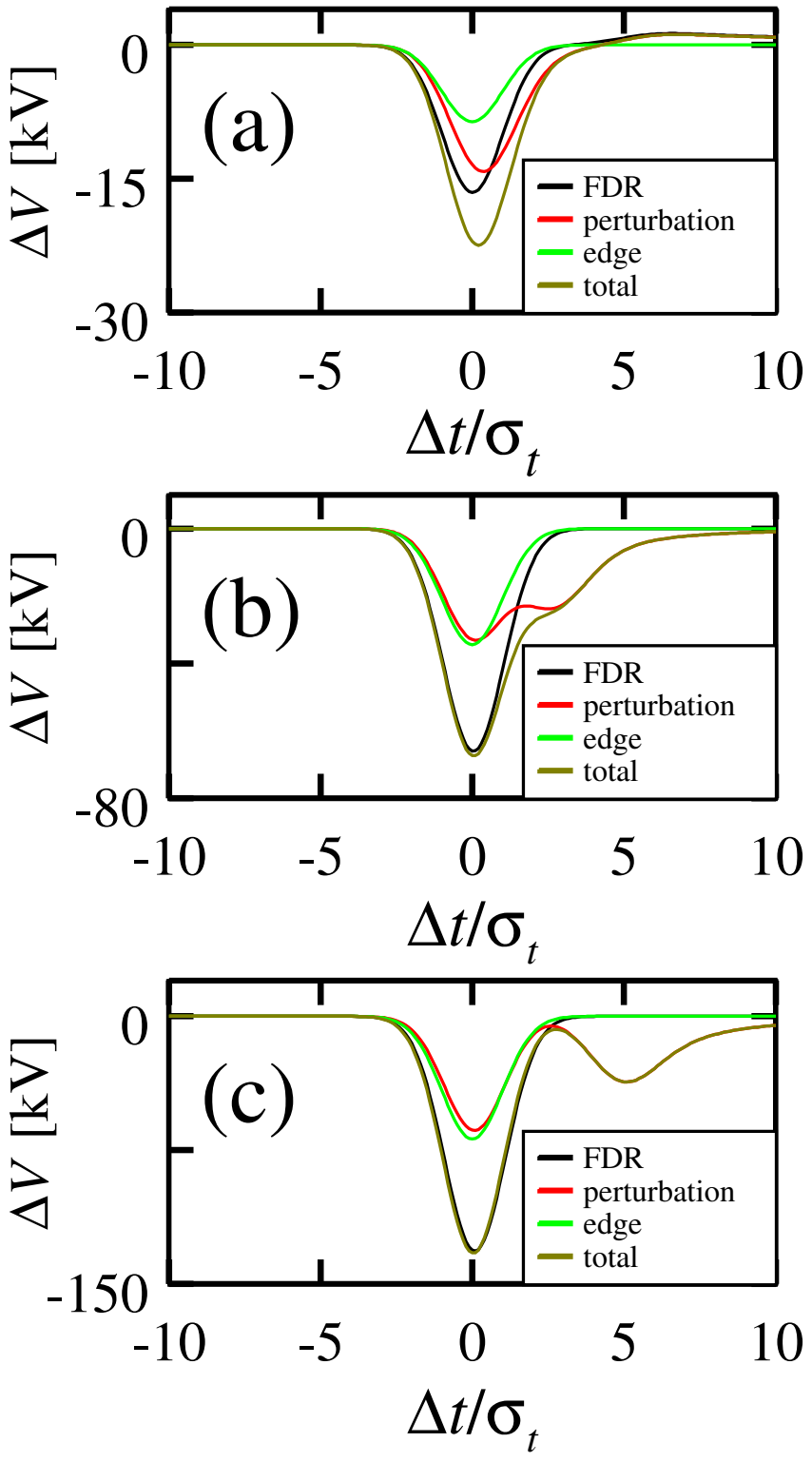

FIG. 5. (Color) Integrated coherent-radiation wakes of a disk target with inner radius of $2 \mathrm{~mm}$ and outer radius of $20 \mathrm{~mm}$, located $1 \mathrm{~m}$ downstream of a bending magnet exit. The wakes are integrated over a distance of $10 \mathrm{~m}$ downstream of the target, for a $511-\mathrm{MeV}, 200-\mathrm{pC}$ Gaussian bunch. We show the edgeradiation wake when there is no target, the perturbation from the target, and the total coherent-radiation wake given by their sum. For comparison, the FDR wake is shown for a target that is far downstream of a bending magnet. (a) $\sigma_{z}=400 \mu \mathrm{m}$. (b) $\sigma_{z}=100 \mu \mathrm{m}$. (c) $\sigma_{z}=50 \mu \mathrm{m}$.

$\mathrm{CDR}$, and the longitudinal wake perturbation is approximated within $20 \%$ by the FDR wake. For the shortest bunch length of $50 \mu \mathrm{m}$, the outer radius exceeds $0.86\left(2 \pi \sigma_{z} L\right)^{1 / 2}=15 \mathrm{~mm}$, and the longitudinal wake perturbation is much different than the FDR wake. In all cases, the small integrated wake perturbation upstream of the target nearly equals the integrated wake of BDR. In these examples, the downstream wake perturbation differs from the FDR wake when a target with outer radius $\geq 0.86\left(2 \pi \sigma_{z} L\right)^{1 / 2}$ intercepts the transverse coherentradiation field of formation-zone edge radiation.

\section{CONCLUSION}

We have calculated the formation-zone longitudinal wakes of a short magnet, transition radiation, and diffraction radiation. Each wake is the sum of a longitudinal space charge wake and a wake of coherent radiation. Our formulas apply within a formation length $\sim \sigma_{z} \gamma^{2}$ of the magnet or target.

For a short magnet, the coherent-radiation wake is a resistive wake from the magnet's exit edge that moves with the bunch, summed with a wake from the magnet's entrance edge that moves ahead of the bunch. For a weak short magnet with deflection angle $\theta \ll 1 / \gamma$, the entranceedge wake moves ahead of the exit-edge wake by a small distance $\ll \sigma_{z}$ within the radiation formation zone, resulting in a steady-state coherent-radiation wake that is much smaller than the bunch's LSC wake. For a strong short magnet with $\theta \gg 1 / \gamma$, the entrance-edge wake overtakes the bunch within the formation zone, where the coherentradiation wake changes from a steady-state wake to the resistive wake of suddenly accelerated electrons $[8,9]$.

By considering an image bunch, we related the wake of transition radiation to that of a deflected bunch. By modeling the diffraction radiation of an iris as the transition radiation of a hollow bunch, we calculated the wake of coherent diffraction radiation for an arbitrary target shape. The additional perturbation when a target is in the formation zone of a bending magnet's coherent edge radiation was also calculated.

Our formulas may be used to evaluate the effects of a short magnet or diffraction-radiation target upon an ultrarelativistic bunch.

\section{ACKNOWLEDGMENTS}

The author thanks K. D. Jacobs, M. A. Green, and J. Wu for useful suggestions, J. J. Bisognano, K. J. Kleman, and R. A. Legg for helpful discussions. This work is based upon research conducted at the Synchrotron Radiation Center, which is supported by National Science Foundation Award No. DMR-0537588 and the University of Wisconsin-Madison.

\section{APPENDIX A: ENTRANCE EDGE RADIATION}

Let the $z$ axis be parallel to the direction of bunch propagation after its deflection through an angle $\theta$. To calculate the wake contribution from an electron that suddenly stops at distance $r$ from the $z$ axis, we use coordinates $\left(x^{\prime}, y^{\prime}, z^{\prime}\right)$, where the electron travels on the $z^{\prime}$ axis before stopping at $\left(x^{\prime}, y^{\prime}, z^{\prime}\right)=(0,0,0)$, as shown in Fig. 6 . 


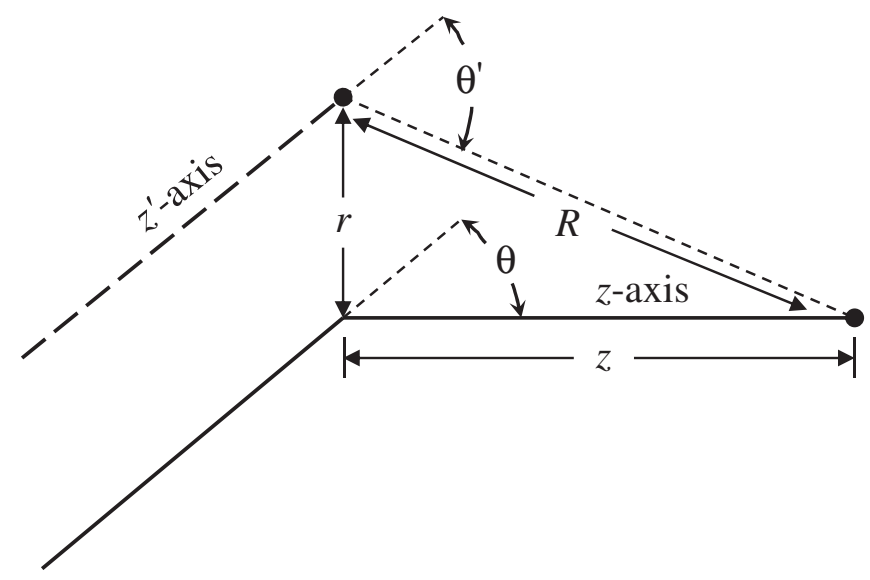

FIG. 6. After being deflected through an angle $\theta$ by a short magnet, the bunch travels parallel to the $z$ axis. To calculate the wake contribution from an electron located at a distance $r$ from the $z$ axis, with azimuthal angle $\phi$, let the $z^{\prime}$ axis describe its path upstream of the magnet. At distance $z$ downstream of the magnet, a point on the $z$ axis is at distance $R$ from the point where the electron is deflected, at polar angle $\theta^{\prime}$ with respect to the $z^{\prime}$ axis.

When an ultrarelativistic electron traveling in uniform motion is suddenly stopped, the radiation is radially polarized. At distance $R \ll \lambda \gamma^{2} / 2 \pi$, the radiation at wavelength $\lambda$ peaks at the polar angle $0.86(\lambda / R)^{1 / 2}$ [12]. When an electron stops in a finite time, emitting radiation over a distance $d \ll \lambda \gamma^{2} / 2 \pi$, the radiation at wavelength $\lambda$ is that of a suddenly stopped electron for $R \gg d$ [12]. The electric field is the sum of the Liénard-Wiechert acceleration and velocity fields [22]. In SI units, the acceleration field of an electron that is stopped at the origin at time $t=0$ is [12]

$$
\begin{aligned}
\tilde{\boldsymbol{E}}_{\boldsymbol{a}}(R, \hat{\boldsymbol{n}}, \omega) & =\int_{-\infty}^{\infty} \boldsymbol{E}_{\boldsymbol{a}}(R, \hat{\boldsymbol{n}}, t) e^{i \omega t} d t \\
& =e^{i k R} \frac{-q}{4 \pi \varepsilon_{0} c R} \frac{\hat{\boldsymbol{n}} \times(\hat{\boldsymbol{n}} \times \boldsymbol{\beta})}{1-\hat{\boldsymbol{n}} \cdot \boldsymbol{\beta}} .
\end{aligned}
$$

Here, $\hat{\boldsymbol{n}}$ is the unit vector pointing from the origin to the observer, and $\boldsymbol{\beta}$ is the electron velocity divided by $c$. For an observer with polar angle $\theta^{\prime}$ with respect to the $z^{\prime}$ axis, we choose the axes so that $y^{\prime}=0$ and $x^{\prime} \geq 0$. In $\left(x^{\prime}, y^{\prime}, z^{\prime}\right)$ coordinates, for $\theta^{\prime} \ll 1$,

$$
\tilde{\boldsymbol{E}}_{\boldsymbol{a}}\left(R, \theta^{\prime}, \omega\right)=\left(\frac{e^{i k R} q}{2 \pi \varepsilon_{0} c R}\right)\left(\frac{-\gamma^{2} \theta^{\prime}}{1+\gamma^{2} \theta^{\prime 2}}, 0, \frac{\gamma^{2} \theta^{\prime 2}}{1+\gamma^{2} \theta^{\prime 2}}\right) .
$$

The velocity field from the electron before it is stopped is [22]

$$
\begin{aligned}
\tilde{\boldsymbol{E}}_{v}\left(\boldsymbol{x}^{\prime}, \omega\right) & =\int_{-\infty}^{\infty} \boldsymbol{E}_{v}\left(\boldsymbol{x}^{\prime}, t_{0}\right) e^{i \omega t_{0}} d t_{0} \\
& =\frac{q}{4 \pi \varepsilon_{0} \gamma^{2}} \int_{-\infty}^{0} \frac{\hat{\boldsymbol{n}}(t)-\boldsymbol{\beta}}{(1-\hat{\boldsymbol{n}}(t) \cdot \boldsymbol{\beta})^{2} R(t)^{2}} e^{i \omega t_{0}(t)} d t .
\end{aligned}
$$

Here, $\hat{\boldsymbol{n}}(t)$ is the unit vector directed from the electron to the observer location $\boldsymbol{x}^{\prime}$, and $R(t)$ is the distance from the electron to the observer. The time $t_{0}$ is the arrival time of radiation emitted at time $t$, obeying $t_{0}(0)=R / c$. Letting $w(t)=\gamma \theta^{\prime}(t)$, where $\theta^{\prime}(t)$ is the angle between $\hat{\boldsymbol{n}}(t)$ and the $z^{\prime}$ axis, Eq. (A3) may be written for $\theta^{\prime} \ll 1$ as [12]

$$
\begin{aligned}
\tilde{\boldsymbol{E}}_{v}\left(R, \theta^{\prime}, \omega\right)= & e^{i k R} \frac{q \gamma e^{i \pi R_{n}\left(1-\varphi^{2}\right)}}{\pi \varepsilon_{0} c R \varphi} \int_{0}^{\varphi} \frac{\left(w, 0, \frac{1-w^{2}}{2 \gamma}\right)}{\left(1+w^{2}\right)^{2}} \\
& \times e^{i \pi R_{n} \varphi(w-1 / w)} d w,
\end{aligned}
$$

where $R_{n} \equiv R / \lambda \gamma^{2}$ and $\varphi \equiv \gamma \theta^{\prime}$. Outside the radiation formation zone $\left(R \gg \lambda \gamma^{2} / 2 \pi\right.$ or $\left.R \theta^{\prime} \gg \lambda \gamma / 2 \pi\right)$, the velocity field vanishes. Within the formation zone $(R \ll$ $\lambda \gamma^{2} / 2 \pi$ and $\left.R \theta^{\prime} \ll \lambda \gamma / 2 \pi\right)$, the approximations $R_{n} \ll$ 1 and $R_{n} \varphi \ll 1$ apply. Approximating the phase term in the integrand of Eq. (A4) as one gives

$$
\begin{aligned}
\tilde{\boldsymbol{E}}_{v}\left(R, \theta^{\prime}, \omega\right) \approx & e^{i k R} \frac{q \gamma e^{-i \pi R_{n} \varphi^{2}}}{\pi \varepsilon_{0} c R \varphi} \\
& \times\left.\left(\frac{w^{2}}{2\left(1+w^{2}\right)}, 0, \frac{w}{2 \gamma\left(1+w^{2}\right)}\right)\right|_{0} ^{\varphi} \\
= & e^{i k R} \frac{q e^{-i k R \theta^{\prime 2} / 2}}{2 \pi \varepsilon_{0} c R\left(1+\gamma^{2} \theta^{\prime 2}\right)}\left(\gamma^{2} \theta^{\prime}, 0,1\right) .
\end{aligned}
$$

Numerical evaluation of Eq. (A4) shows that Eq. (A5) describes the transverse field for $R_{n} \ll 1$ and $R_{n} \varphi \ll 1$, i.e. $R \ll \lambda \gamma^{2} / 2 \pi$ and $R \theta^{\prime} \ll \lambda \gamma / 2 \pi$. The expression for the axial field is accurate within a smaller region $\left[R_{n} \ll 1\right.$ and $R_{n} \varphi^{2} \ll 1$, i.e. $R \ll \lambda \gamma^{2} / 2 \pi$ and $R \theta^{\prime} \ll(\lambda R)^{1 / 2}$ ], where the phase $\exp \left(-i k R \theta^{12} / 2\right)$ is approximately one $[9,12]$.

Summing the velocity and acceleration fields gives the electric field

$$
\tilde{\boldsymbol{E}}_{\mathrm{ent}}\left(R, \theta^{\prime}, \omega\right)=\left(\frac{e^{i k R} q}{2 \pi \varepsilon_{0} c R}\right)\left(\frac{\gamma^{2} \theta^{\prime}\left(e^{-i k R \theta^{\prime 2} / 2}-1\right)}{1+\gamma^{2} \theta^{\prime 2}}, 0,1\right)
$$

for $R \ll \lambda \gamma^{2} / 2 \pi, R \theta^{\prime} \ll \lambda \gamma / 2 \pi$, and $\theta^{\prime} \ll 1$.

Downstream of the magnet, the longitudinal field from the electron's entrance edge radiation is $\tilde{\boldsymbol{E}}_{\text {ent }}\left(R, \theta^{\prime}, \omega\right) \cdot \hat{z}$. For $R \gg r, \hat{z} \approx(\sin \theta, 0, \cos \theta)$ in $\left(x^{\prime}, y^{\prime}, z^{\prime}\right)$ coordinates, while $\theta^{\prime} \approx \theta+r \cos \phi / R$ for an electron with azimuthal angle $\phi$. For $\theta \ll 1$, approximating $\hat{z} \approx(\theta, 0,1)$ gives 
$\tilde{\boldsymbol{E}}_{\mathrm{ent}}\left(R, \theta^{\prime}, \omega\right) \cdot \hat{z}=\left(\frac{e^{i k R} q}{2 \pi \varepsilon_{0} c R}\right)\left(1+\frac{\gamma^{2} \theta^{\prime} \theta\left(e^{-i k R \theta^{\prime 2} / 2}-1\right)}{1+\gamma^{2} \theta^{\prime 2}}\right)$

For $|r \cos \phi / R| \ll \theta$, setting $\theta^{\prime}=\theta$ in Eq. (A7) gives $\tilde{\boldsymbol{E}}_{\mathrm{ent}} \cdot \hat{\boldsymbol{z}} \approx e^{i k R} e^{-i k R \theta^{2} / 2} q / 2 \pi \varepsilon_{0} c R$.

For $|r \cos \phi / R| \gg \theta,\left|\theta / \theta^{\prime}\right| \ll 1$ implies that $\tilde{\boldsymbol{E}}_{\mathrm{ent}} \cdot \hat{\boldsymbol{z}} \approx$ $e^{i k R} q / 2 \pi \varepsilon_{0} c R$. For bunch length $\sigma_{z}$, the profile is dominated by wave numbers $k \leq 1 / \sigma_{z}$, and the wake contribution from radius $r$ affects the bunch at distances $R \gtrsim \max \left(r, r^{2} / \sigma_{z}\right) \quad$ [9], where $e^{i k R} q / 2 \pi \varepsilon_{0} c R \approx$ $e^{i k R} e^{-i k R \theta^{2} / 2} q / 2 \pi \varepsilon_{0} c R$. For $|r \cos \phi / R| \sim \theta$, we have $\tilde{\boldsymbol{E}}_{\text {ent }} \cdot \hat{z} \approx e^{i k R} e^{-i k R \theta \theta^{\prime} / 2} q / 2 \pi \varepsilon_{0} c R$, which is also approximated by $e^{i k R} e^{-i k R \theta^{2} / 2} q / 2 \pi \varepsilon_{0} c R$ for $R \gtrsim \max \left(r, r^{2} / \sigma_{z}\right)$. Thus, for $\theta^{\prime} \ll 1$, we approximate

$$
\tilde{\boldsymbol{E}}_{\mathrm{ent}}\left(R, \theta^{\prime}, \omega\right) \cdot \hat{z} \approx \frac{e^{i k R} e^{-i k R \theta^{2} / 2} q}{2 \pi \varepsilon_{0} c R} \approx \frac{e^{i k R \cos \theta} q}{2 \pi \varepsilon_{0} c R}
$$

For $\theta^{\prime} \gg 1 / \gamma$, the velocity field is approximated by integrating Eq. (A3) over $-\infty<t<\infty$, since the contribution from $0<t<\infty$ is small [20]. This gives the Coulomb field of an electron in uniform motion [10], in $\left(x^{\prime}, y^{\prime}, z^{\prime}\right)$ coordinates,

$$
\begin{aligned}
\tilde{\boldsymbol{E}}_{\text {Coul }}\left(R, \theta^{\prime}, \omega\right)= & \frac{e^{i \omega z^{\prime} / \beta c} q}{2 \pi \varepsilon_{0} \gamma^{2} \beta^{2} c^{2}} \\
& \times\left(|\omega| \gamma K_{1}\left(\frac{|\omega| x^{\prime}}{\beta c \gamma}\right), 0,-i \omega K_{0}\left(\frac{|\omega| x^{\prime}}{\beta c \gamma}\right)\right) \\
\approx & \frac{e^{i k z \cos \theta} q}{2 \pi \varepsilon_{0} c R \sin \theta^{\prime}} \\
& \times\left(1,0, \frac{-i \omega R \sin \theta^{\prime}}{c \gamma^{2}} K_{0}\left(\frac{|\omega| R \sin \theta^{\prime}}{c \gamma}\right)\right) .
\end{aligned}
$$

The approximate expression applies within the formation zone, where $\beta \approx 1$ and $K_{1}(x) \approx 1 / x$. For $\theta^{\prime} \gg 1 / \gamma$, $\tilde{\boldsymbol{E}}_{\mathrm{ent}}\left(R, \theta^{\prime}, \omega\right)=\tilde{\boldsymbol{E}}_{\text {Coul }}\left(R, \theta^{\prime}, \omega\right)+\tilde{\boldsymbol{E}}_{a}\left(R, \theta^{\prime}, \omega\right)$, where

$$
\begin{aligned}
\tilde{\boldsymbol{E}}_{\mathrm{Coul}}\left(R, \theta^{\prime}, \omega\right) \cdot \hat{z}= & \frac{e^{i k z \cos \theta} q}{2 \pi \varepsilon_{0} c R}\left[\frac{\sin \theta}{\sin \theta^{\prime}}\right. \\
& \left.-\frac{i \omega R \cos \theta}{c \gamma^{2}} K_{0}\left(\frac{|\omega| R \sin \theta^{\prime}}{c \gamma}\right)\right]
\end{aligned}
$$

and

$$
\tilde{\boldsymbol{E}}_{a}\left(R, \theta^{\prime}, \omega\right) \cdot \hat{z}=\frac{e^{i k R} q \beta \sin \theta^{\prime} \sin \left(\theta^{\prime}-\theta\right)}{4 \pi \varepsilon_{0} c R\left(1-\beta \cos \theta^{\prime}\right)} .
$$

For $R \gg r / \theta$ [i.e., $z \sin (\theta / 2) \gg r$ ], noting that $\sin \left(\theta^{\prime}-\right.$ $\theta)=r \cos \phi / R$ gives $\left|\tilde{\boldsymbol{E}}_{a}\left(R, \theta^{\prime}, \omega\right) \cdot \hat{z}\right| \ll\left|q / 2 \pi \varepsilon_{0} c R\right|$, indicating that $\tilde{\boldsymbol{E}}_{\mathrm{ent}}\left(R, \theta^{\prime}, \omega\right) \cdot \hat{z} \approx \tilde{\boldsymbol{E}}_{\mathrm{Coul}}\left(R, \theta^{\prime}, \omega\right) \cdot \hat{z}$.
When $R \gg r / \sin \theta$ and $\sin \theta \gg 1 / \gamma$ are obeyed, $\sin \theta^{\prime} \approx \sin \theta$ and

$$
\tilde{\boldsymbol{E}}_{\mathrm{Coul}}\left(R, \theta^{\prime}, \omega\right) \cdot \hat{z} \approx \frac{e^{i k z \cos \theta} q}{2 \pi \varepsilon_{0} c R}
$$

Equations (A8) and (A12) indicate that, for $0 \leq \theta \ll \pi-$ $1 / \gamma$

$$
\tilde{\boldsymbol{E}}_{\mathrm{ent}}\left(R, \theta^{\prime}, \omega\right) \cdot \hat{z} \approx \frac{e^{i k R \cos \theta} q}{2 \pi \varepsilon_{0} c R}
$$

where $R=\sqrt{z^{2}+r^{2}}$. Equation (A13) applies for $z \gg r$ when $\theta \leq \pi / 2$. For $\pi / 2<\theta \ll \pi-1 / \gamma$, Eq. (A13) applies where $z \gg r / \sin \theta$ : when the observer is not in the region occupied by the bunch while upstream of the magnet. The conditions for applicability of Eq. (A13) may be written as $(\pi-\theta) / 2 \gg 1 / \gamma$ and $z \sin [(\pi-\theta) / 2] \gg r$.

\section{APPENDIX B: COHERENT DIFFRACTION RADIATION FROM AN IRIS}

Consider the Kirchhoff diffraction integral for the longitudinal electric field when an on-axis ultrarelativistic electron passes through a circular iris of radius $a$ at time $t=0$. The frequency-domain field is integrated over the target to obtain the coherent-radiation field of an on-axis downstream observer,

$$
\begin{aligned}
\tilde{E}(z, \omega) & \equiv \int_{-\infty}^{\infty} E(z, t) e^{i \omega t} d t \\
& =(k / 2 \pi i) \int_{0}^{2 \pi} d \phi \int_{a}^{\infty} r d r \tilde{E}(r, \phi, \omega) e^{i k D} / D,
\end{aligned}
$$

where $D=\sqrt{z^{2}+r^{2}}$ is the distance from a point on the target to the observer. Letting $\tilde{E}(r, \phi, \omega)=$ $\left(-i q \omega / 2 \pi \varepsilon_{0} c^{2} \gamma^{2}\right) K_{0}(|\omega| r / c \gamma)$ be the longitudinal Coulomb field gives

$$
\tilde{E}(z, \omega)=\frac{-q \omega^{2}}{2 \pi \varepsilon_{0} c^{3} \gamma^{2}} \int_{a}^{\infty} r d r K_{0}\left(\frac{|\omega| r}{c \gamma}\right) \frac{e^{i k \sqrt{z^{2}+r^{2}}}}{\sqrt{z^{2}+r^{2}}}
$$

For $z \ll \sigma_{z} \gamma^{2}$, the integrand's phase oscillates where $r>$ $\lambda \gamma / 2 \pi$. Assuming that the integral is dominated by the nearest target regions, setting $\sqrt{z^{2}+r^{2}} \approx \sqrt{z^{2}+a^{2}}$ gives

$$
\tilde{E}(z, \omega) \approx \frac{-q \omega^{2}}{2 \pi \varepsilon_{0} c^{3} \gamma^{2}} \frac{e^{i k \sqrt{z^{2}+a^{2}}}}{\sqrt{z^{2}+a^{2}}} \int_{a}^{\infty} r d r K_{0}\left(\frac{|\omega| r}{c \gamma}\right)
$$

For a target with $a \ll \sigma_{z} \gamma$, extending the lower limit of integration to $r=0$ gives [23] 


$$
\begin{aligned}
\tilde{E}(z, \omega) & \approx \frac{-q \omega^{2} e^{i k \sqrt{z^{2}+a^{2}}}}{2 \pi \varepsilon_{0} c^{3} \gamma^{2} \sqrt{z^{2}+a^{2}}} \int_{0}^{\infty} r d r K_{0}\left(\frac{|\omega| r}{c \gamma}\right) \\
& =\left(\frac{-q}{2 \pi \varepsilon_{0} c}\right) \frac{e^{i k \sqrt{z^{2}+a^{2}}}}{\sqrt{z^{2}+a^{2}}} .
\end{aligned}
$$

The on-axis wake from a bunch of on-axis electrons is

$$
\begin{aligned}
W_{\text {iris }}(z, \Delta t) & =(N / 2 \pi) \int_{-\infty}^{\infty} F(\omega) \tilde{E}(z, \omega) e^{-i k z-i \omega \Delta t} d \omega \\
& \approx\left(\frac{-N q}{2 \pi \varepsilon_{0} c \sqrt{z^{2}+a^{2}}}\right) f\left(\Delta t-\frac{\sqrt{z^{2}+a^{2}}-z}{c}\right) .
\end{aligned}
$$

[1] J. B. Murphy, S. Krinsky, and R. L. Gluckstern, Part. Accel. 57, 9 (1997).

[2] E. L. Saldin, E. A. Schneidmiller, and M. V. Yurkov, Nucl. Instrum. Methods Phys. Res., Sect. A 398, 373 (1997).

[3] G. Stupakov and P. Emma, in Proceedings of the Eighth European Particle Accelerator Conference, Paris (EPSIGA/CERN, Geneva, 2002), p. 1479.

[4] M. Borland, Y. C. Chae, P. Emma, J. W. Lewellen, V. Bharadwaj, W. M. Fawley, P. Krejcik, C. Limborg, S. V. Milton, H.-D. Nuhn, R. Soliday, and M. Woodley, Nucl. Instrum. Methods Phys. Res., Sect. A 483, 268 (2002).

[5] D. Davino, G. Miano, G. Panariello, and L. Verolino, Phys. Rev. ST Accel. Beams 2, 044401 (1999).

[6] K.L.F. Bane and G. Stupakov, Phys. Rev. ST Accel. Beams 7, 064401 (2004).

[7] Dao Xiang, Wen-Hui Huang, Yu-Zheng Lin, Sung-ju Park, and In Soo Ko, Phys. Rev. ST Accel. Beams 11, 024001 (2008).
[8] G. Geloni, E. Saldin, E. Schneidmiller, and M. Yurkov, Nucl. Instrum. Methods Phys. Res., Sect. A 578, 34 (2007).

[9] R. A. Bosch, Phys. Rev. ST Accel. Beams 10, 050701 (2007).

[10] J.D. Jackson, Classical Electrodynamics (Wiley, New York, 1975), 2nd ed., p. 688, Eq. 14.125.

[11] R. A. Bosch, Nucl. Instrum. Methods Phys. Res., Sect. A 386, 525 (1997).

[12] R. A. Bosch and O. V. Chubar, in Synchrotron Radiation Instrumentation, edited by E. Fontes, AIP Conf. Proc. No. 417 (AIP, New York, 1997), p. 35.

[13] M. Castellano, in Proceedings of the 1999 Particle Accelerator Conference, New York (IEEE, Piscataway, NJ, 1999), p. 2695.

[14] R. Coïsson, Phys. Rev. A 20, 524 (1979).

[15] J. J. Bisognano, R. A. Bosch, M. A. Green, K. D. Jacobs, K. J. Kleman, R. A. Legg, J. Chen, W. S. Graves, F. X. Kärtner, and J. Kim, in Proceedings of the 2007 Particle Accelerator Conference, Albuquerque, NM (IEEE, Piscataway, NJ, 2007), p. 1281.

[16] A. P. Potylitsyn, Nucl. Instrum. Methods Phys. Res., Sect. B 145, 169 (1998).

[17] D. Sütterlin, D. Erni, M. Dehler, H. Jäckel, H. Sigg, and V. Schlott, Nucl. Instrum. Methods Phys. Res., Sect. B 264, 361 (2007).

[18] D. V. Karlovets and A. P. Potylitsyn, Nucl. Instrum. Methods Phys. Res., Sect. B 266, 3738 (2008).

[19] S. A. Kheifets, IEEE Trans. Microwave Theory Tech. 35, 753 (1987).

[20] R. A. Bosch, Rev. Sci. Instrum. 73, 1423 (2002).

[21] P. Roy, Y.-L. Mathis, A. Paolone, P. Giura, A. Nucara, S. Lupi, P. Calvani, and A. Gerschel, Nuovo Cimento Soc. Ital. Fis. D 20, 415 (1998).

[22] J.D. Jackson, Classical Electrodynamics (Wiley, New York, 1975), 2nd ed., pp. 657, 670.

[23] I. S. Gradshteyn and I. M. Ryzhik, Table of Integrals, Series, and Products (Academic Press, San Diego, 1994), 5th ed., p. 708, Eq. 6.561.16. 